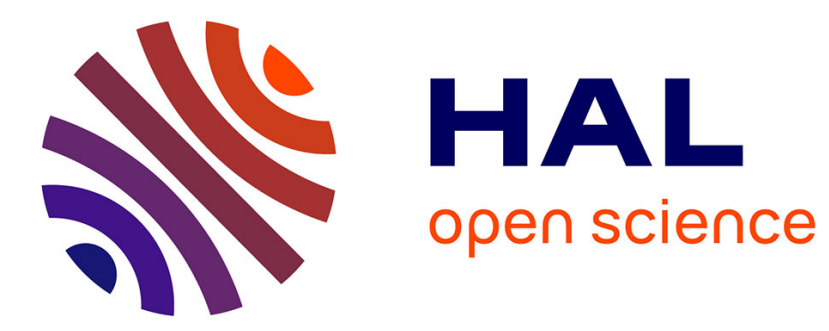

\title{
FTIR techniques applied to the detection of gelatine in paper artifacts: from macroscopic to microscopic approach
}

\author{
Véronique Rouchon, E Pellizzi, K Janssens
}

\section{- To cite this version:}

Véronique Rouchon, E Pellizzi, K Janssens. FTIR techniques applied to the detection of gelatine in paper artifacts: from macroscopic to microscopic approach. Applied physics. A, Materials science \& processing, 2010, 100 (3), pp.663-669. 10.1007/s00339-010-5649-5 . hal-01469702

\author{
HAL Id: hal-01469702 \\ https://hal.science/hal-01469702
}

Submitted on 16 Feb 2017

HAL is a multi-disciplinary open access archive for the deposit and dissemination of scientific research documents, whether they are published or not. The documents may come from teaching and research institutions in France or abroad, or from public or private research centers.
L'archive ouverte pluridisciplinaire HAL, est destinée au dépôt et à la diffusion de documents scientifiques de niveau recherche, publiés ou non, émanant des établissements d'enseignement et de recherche français ou étrangers, des laboratoires publics ou privés. 


\title{
FTIR techniques applied to the detection of gelatine in paper artifacts: from macroscopic to microscopic approach
}

\author{
Véronique Rouchon · Eleonora Pellizzi · Koen Janssens
}

Received: 28 August 2009 / Accepted: 9 March 2010 / Published online: 2 April 2010

(C) Springer-Verlag 2010

\begin{abstract}
In order to render paper hydrophobic for ink and thus adequate for writing, gelatine has been largely used. To this day, it is still employed in conservation workshops as an adhesive or a sizing agent, for instance, during the treatment of iron gall ink manuscripts. Various types and concentrations of gelatine are recommended, depending on the desired effect, but little information is available regarding to the physical distribution of gelatine in the paper. This aspect is however determinant for a better control of conservation treatments.

In this work, we investigate the possibilities offered by FTIR microscopy for the measurement of the gelatine distribution in paper. Laboratory papers were preliminary treated with different types of gelatine and then embedded in a resin and cut in thin slices. Mapping techniques enable to compare the penetration of different types of gelatine in a semiquantitative way. The performance of conventional laboratory equipment and synchrotron radiation experimental setup are discussed.
\end{abstract}

\footnotetext{
V. Rouchon $(\bowtie) \cdot$ E. Pellizzi

Centre de Recherche sur la Conservation des Collections, Muséum National d'Histoire Naturelle, URS 3224,

MNHN-CNRS-MCC, 36 rue Saint Hilaire, 75005 Paris, France e-mail: rouchon@mnhn.fr

Fax: +33-1-40795312

E. Pellizzi

e-mail: pellizzi@mnhn.fr

\section{K. Janssens}

Centre for Micro- and Trace Analysis, Department of Chemistry, Universiteit Antwerpen, Universiteitsplein 1, 2610 Antwerp,

Belgium

e-mail: koen.janssens@ua.ac.be

Fax: +32-3-8202376
}

\section{Introduction}

Traditionally, occidental paper making used collagen derivatives in order to render paper hydrophobic for ink and thus adequate for writing. The introduction of gelatine sizing is generally attributed to the Italian paper mills of Fabriano. These mills became one of the predominant paper manufacturing centers at the end of the thirteenth century [1]. As little analytical data is available regarding the composition of early European paper, it is not known in detail how the type of size was discovered, how it evolved, or how it became adopted on a large scale in the fifteenth century.

The gelatine analysis in paper appears technically possible: gelatine seems to be a rather stable paper component. Stephen et al. [2] detected, on a set of nine specimens dating from the fifteenth century, large amounts of gelatine (over $4.5 \% \mathrm{w} / \mathrm{w}$ ) still remaining in the paper. Many analytical techniques have been employed for detecting gelatine or determining its content in paper [3]: colorimetric spot tests [4], detection of amino acids by gas chromatography-mass spectrometry [2] or by spectrophotometry [5, 6], molecular fluorescence measurements [7], ultrasonic measurements [8] and FTIR spectrometry [8].

Yet, only a few of these techniques yield quantitative reliable measurements. These are usually achieved on the basis of calibration data obtained from modern papers impregnated with different amounts of gelatine. The interpretation of these quantitative measurements presents two points that merit discussion. Firstly, as the degradation of gelatine versus time is not well known, it remains difficult to relate the actual observed amount of gelatine to a supposed original amount. Amino acids, such as hydroxyproline, are believed to be stable, but it remains evident that gelatine may degrade over a period of several centuries. Size exclusion chromatography measurements performed on artificially aged 
gelatine sized papers point to a significant depolymerization of gelatine $[9,10]$. Similarly, optical measurements, performed on artificially aged, gelatine sized papers showed a significant yellowing with aging that is attributed to the presence of gelatine in the paper [11]. Secondly, the gelatine content measurements are related to average values that do not take into account the size distribution within the paper thickness. This distribution, however, may have an impact on some of the techniques used for gelatine measurements, such as infrared spectrometry. It may additionally give some valuable information on the sizing procedure.

Despite all these uncertainties, some recurrent observations were formulated regarding the gelatine content and the paper conservation state: conservation is better when the presence of gelatine is found to be more important $[1,12]$. The $\mathrm{pH}$ of the paper is an additional determining parameter, depending on the amount of charge present in the paper (calcium carbonate and magnesium carbonate), and on the presence of alum, used to induce gelatine precipitation.

Today, gelatine is being replaced by synthetic sizes, and its use in paper making is limited to some specific quality papers for artists. Its main application in relation to paper concerns conservation treatments: it is, in north European countries, one of the favorite adhesives used to repair or resize original papers. Various types and concentrations of gelatine are advised [13]. On iron gall ink manuscripts for instance, Gelatine type B, 220 bloom, is preferred as it is supposed to "fix" iron and prevent its migration during the application [14]. Here again, the depth distribution of gelatine, when applied on an original sheet of paper, remains unknown. This aspect is however determinant for a better control of conservation treatments.

FTIR spectroscopy has been frequently used for protein analysis $[15,16]$. The Amide I band, near $1650 \mathrm{~cm}^{-1}$, is associated with $\mathrm{C}=\mathrm{O}$ stretching vibration of peptide groups and presents several frequencies that depend upon the chemical environment and consequently upon the conformational state of polypeptides. The Amide II band, near $1550 \mathrm{~cm}^{-1}$ is associated with $\mathrm{C}-\mathrm{N}$ stretching and $\mathrm{N}-\mathrm{H}$ bending vibrations of peptide groups. These two bands are usually considered to be valuable indicators of the presence of gelatine in original paper [17]. Determination of gelatine content in paper with infrared spectroscopy, in Attenuated Total Reflectance (ATR) and diffuse reflectance mode, was also attempted $[3,7,8]$, but only few data are published, and the calibration curves appeared to be of limited reliability [3]. However, one may expect that the spectral area of the amide I and amide II band could constitute valuable indicators of the gelatine content, providing that this later is not degraded.

In this work, we investigate the possibilities offered by FTIR for the measurement of the gelatine distribution in paper. Samples, consisting of paper impregnated with different types of gelatine, are first examined in ATR mode.
This macroscopic and surface sensitive approach depends on the, possibly uneven, distribution of gelatine throughout the thickness of the paper. This last point seriously limits the possibilities for quantitative determination. When used for characterization of thinly sliced, resin-embedded samples in transmission geometry, FTIR microscopy proved itself to be a suitable analytical technique with imaging capabilities. The microscopic approach enables the measurement of the gelatine gradient throughout the paper, providing valuable additional information.

\section{Experimental}

\subsection{Sample preparation}

\subsubsection{Laboratory samples}

Laboratory samples were prepared in order to define the relevant parameters to be used in order to make the technique semiquantitative. They consist in Whatman No. 1 filter paper that is impregnated with two types of gelatine previously used in Dupont's work [9]: a pharmaceutical/food grade type A gelatine, called "N" and produced by Norland from acid treated fish skin (Batch 7345 HMWD), and a photographic grade type B gelatine, called " $\mathrm{K}$ " and produced by Kind and Knox from alkali treated cattle bones (Gelita Type 8039).

The impregnation was performed by immersing the paper for 1 minute in the gelatine solution maintained at $40^{\circ} \mathrm{C}$ $\pm 0.5^{\circ} \mathrm{C}$, using different concentrations of gelatine, ranging from $0.25 \%$ to $6 \%$. This concentration range is similar to that tested by Barrett et al. [4], and it is believed to be representative of the possible concentrations used during paper making. After immersion, the samples were hung vertically, and the excess gelatine solution was removed by capillarity action on the paper edge using a blotting paper placed perpendicular to the sample. No alum and no artificial aging procedures were used.

The average gelatine uptake was evaluated by weighing the samples just before and after the gelatine bath. It was similar for both types of gelatine used (Fig. 1), despite the fact that for the same concentration, the " $\mathrm{K}$ " type gelatine solution was obviously more viscous than " $\mathrm{N}$ " type.

\subsubsection{Preparation of micro slices}

The preparation of micro slices was necessary to map the gelatine distribution in the paper. Preliminary tests showed that the thickness of these slices should remain largely below $5 \mu \mathrm{m}$ in order to avoid excessive absorption of the primary IR radiation. The embedding of the samples in resin largely facilitates the preparation of such slices. Several commercial resins were tested. We finally choose the viscosity Embedding kit from Electron Microscopy Sciences (reference 


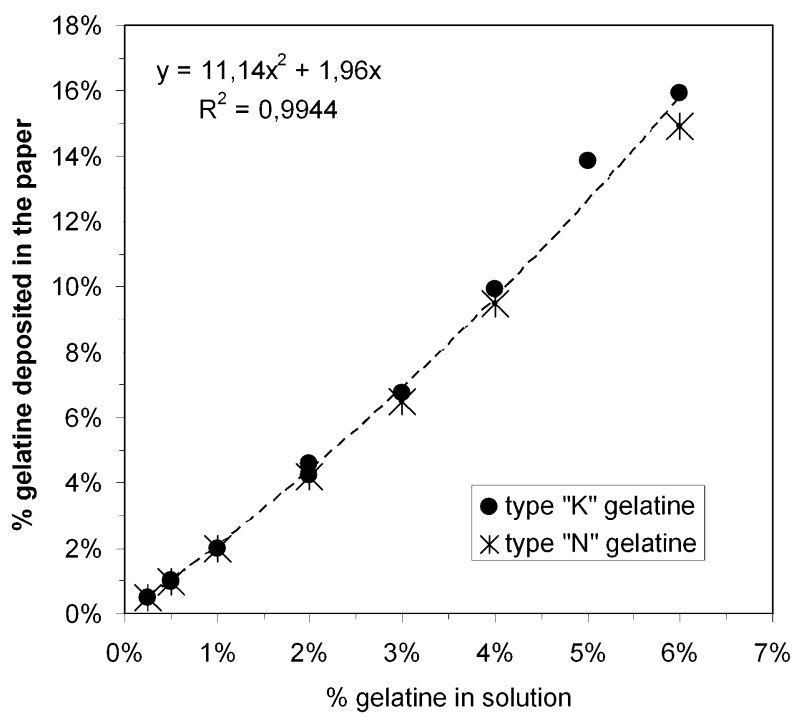

Fig. 1 Gelatine uptake in the paper versus its concentration in solution. Uptakes (expressed in percentage w/w of dry mater), evaluated by weight measurements before and after impregnation, are very similar for both kinds of gelatine. The dotted line corresponds to a second-order polynomial interpolation of the data

14300, reactant proportions: $10 \mathrm{ml}$ of ERL 4221, $6 \mathrm{ml}$ of DER 736, $23 \mathrm{ml}$ of NSA, and $0.4 \mathrm{ml}$ of DMAE) for several reasons. Firstly, in the IR wavenumber domain of this work $\left(1700 \mathrm{~cm}^{-1}\right.$ to $\left.1500 \mathrm{~cm}^{-1}\right)$, the absorption rate is limited (Fig. 2). Secondly, the resin does not dissolve gelatine. Thirdly, it can polymerize below $40^{\circ} \mathrm{C}$, thus avoiding any thermal degradation to gelatine or paper. To remove water and thus facilitate the penetration of the resin in the paper, all samples were kept overnight in a vacuum chamber before embedding. The polymerization of the resin was realized at $37^{\circ} \mathrm{C}$ for approximately five days. One or two micron thick slices were then prepared using a diamond knife (type TS-S-55 ${ }^{\circ}$ from Microstar Technologies) and an ultramicrotome (Ultracut E from Reichert-Jung). Two types of slices were prepared: the first (transversal slices), 1 to $1.5 \mu \mathrm{m}$ thick, was cut perpendicular to the plane of the paper, in order to identify qualitatively the gelatine distribution in the paper. The second (parallel slices), $2-\mu \mathrm{m}$ thick, was cut parallel to the paper plane. These slides were cut progressively along the paper thickness of each sample and were then stuck with a glue pencil (grid coated pen from Electron Microscopy Sciences) on a 0.9 mm-thick $\mathrm{CaF}_{2}$ carrier.

\subsection{FTIR measurements}

All maps and spectra were processed by means of the OMNIC 7.3 software provided by Thermo Electron.

\subsubsection{ATR-FTIR measurements}

In order to evaluate the possibility of ATR-FTIR measurements for rapid detection of gelatine in paper, we first used a Nicolet 6700 Spectrophotometer, equipped with a diamond ATR macro-system (Smart Endurance). Measurements were performed on laboratory samples directly on the paper surface. They consist in evaluating the respective areas of the amide I $\left(1720 \mathrm{~cm}^{-1}\right.$ to $\left.1590 \mathrm{~cm}^{-1}\right)$ and amide II $\left(1590 \mathrm{~cm}^{-1}\right.$ to approximately $1500 \mathrm{~cm}^{-1}$ ) absorption after the subtraction of the linear background between $1720 \mathrm{~cm}^{-1}$ and approximately $1500 \mathrm{~cm}^{-1}$ (Fig. 2). Most of the samples were rather homogeneous. Standard deviations were low, and an average of 5 measurements was considered to be representative for each sample. On the samples impregnated with a high concentration of gelatine "K", which were very heterogeneous, the average of 15 to 20 measurements was calculated.

\subsubsection{FTIR microscopy}

In order to evaluate the distribution of gelatine in the paper, FTIR mappings were performed on micro slices in transmission geometry and using two similar FTIR Microscopes (Nicolet Continuum XL) from Thermo Electron Corporation, equipped with Nicolet 6700 Spectrophotometers. The first uses the conventional laser source provided with the equipment. The second is specifically adapted to a synchrotron radiation source (SOLEIL, SMIS line, France). The synchrotron radiation presents a much higher intensity than a laser source, thus minimizing the background and enabling the mapping of a sample with an improved spatial resolution compared to conventional equipment. On the samples studied here, using a beam aperture of $20 \mu \mathrm{m}$ was necessary in order to obtain a satisfactory signal/noise ratio by means of the conventional equipment. On the synchrotron radiation, this aperture could easily be lowered down to $8 \mu \mathrm{m}$.

As the thickness of the paper is close to hundred micrometers, it was found more appropriate to examine the transversal slices on the synchrotron experimental setup. Maps were then recorded using the following parameters: $\times 32$ objective, $10 \mu \mathrm{m} \times 10 \mu \mathrm{m}$ beam aperture, $10 \mu \mathrm{m}$ steps, gain 1,20 scans (spectra), 64 scans (background), $8 \mathrm{~cm}^{-1}$ resolution, and $4000 \mathrm{~cm}^{-1}$ to $800 \mathrm{~cm}^{-1}$ energy range.

No optimized spatial resolution was necessary for the examination of the lateral slices: their composition was supposed to be rather uniform, and only average values were sought. On these slices, maps were also recorded with the conventional equipment, scanning a large area with a low spatial resolution and using the following parameters: $\times 15$ objective, $90 \mu \mathrm{m} \times 90 \mu \mathrm{m}$ beam aperture, $60 \mu$ steps, gain 1 , 64 scans, $8 \mathrm{~cm}^{-1}$ resolution, and $4000 \mathrm{~cm}^{-1}$ to $800 \mathrm{~cm}^{-1}$ energy range. 
Fig. 2 Some examples of ATR-FTIR spectra. The resin and the glue used in the preparation of the sample show little absorption in the region of interest for the study of gelatine $\left(1500-1700 \mathrm{~cm}^{-1}\right)$. Whatman paper respectively impregnated with "K" type and "N" type gelatines show very different profiles, despite similar gelatine contents

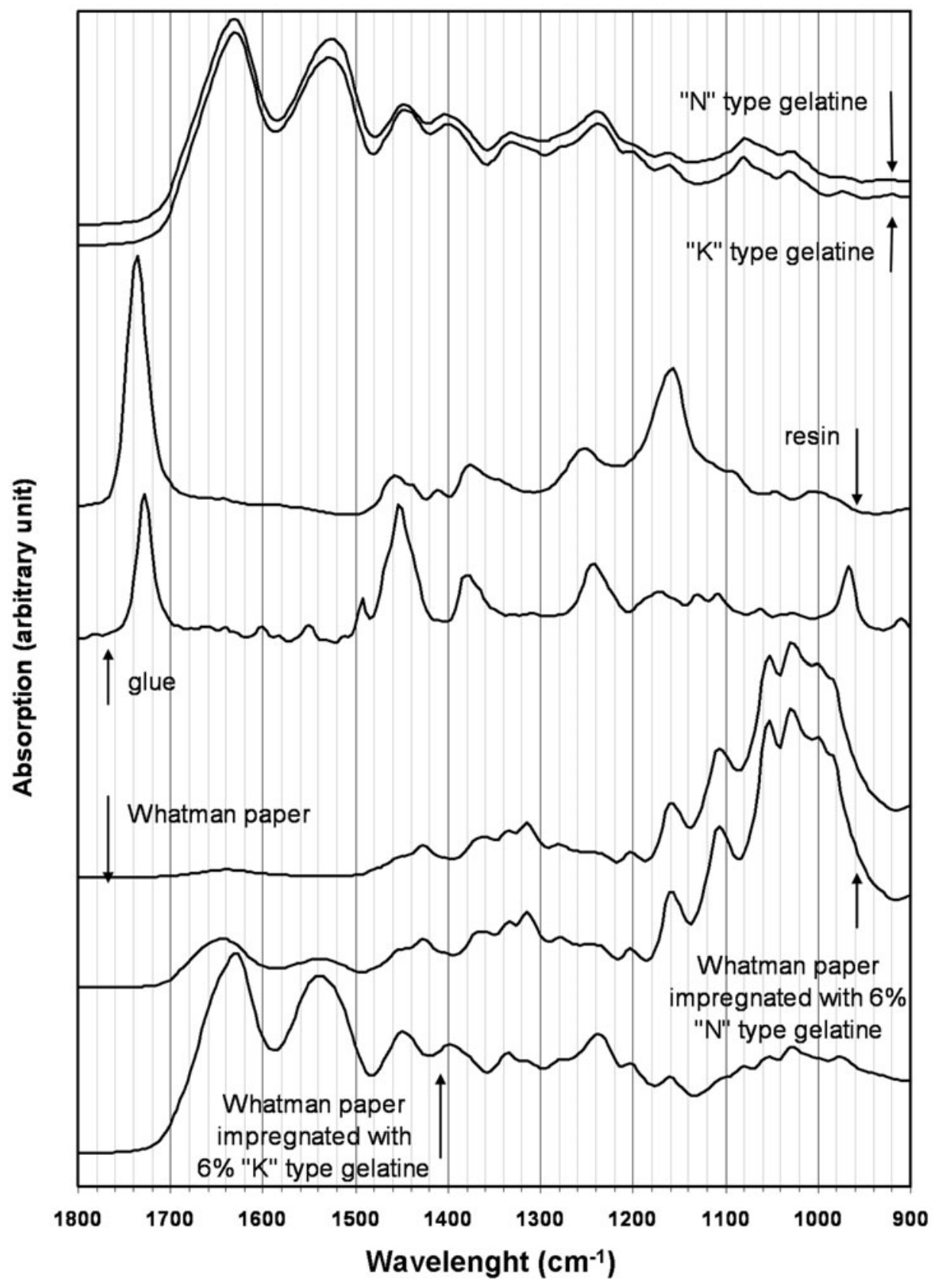

\section{Results}

\subsection{ATR-FTIR measurements}

Despite the fact that the average contents are very similar for the two types of gelatine (Fig. 1), we obtained very different ATR responses (Fig. 3). On "N" type samples, the amide I and amide II bands increase regularly and linearly with the average gelatine content. On " $\mathrm{K}$ " type samples, the intensities of the amide I and amide II bands are much higher. They increase more or less linearly between $2 \%$ w/w and $10 \% \mathrm{w} / \mathrm{w}$ of the gelatine deposition and remain constant over this limit. Consequently, the IR spectra of gelatine-rich samples vary considerably from one type of gelatine to another. On the sample impregnated with $6 \%$ " $\mathrm{K}$ " type gelatine solution, the amide bands largely dominate the cellulose absorption. On the sample impregnated with $6 \%$ "N" type gelatine, amide and cellulose absorption peaks of similar intensity can be observed (Fig. 2).

These observations show that the size repartition within the paper thickness is different for the two gelatines: "N" type is less present at the paper surface than " $\mathrm{K}$ " type. We also conclude that " $\mathrm{N}$ " type penetrates more deeply in the paper than " $K$ " type. The difference in fluidity for the same concentration may be a possible explanation for this. 

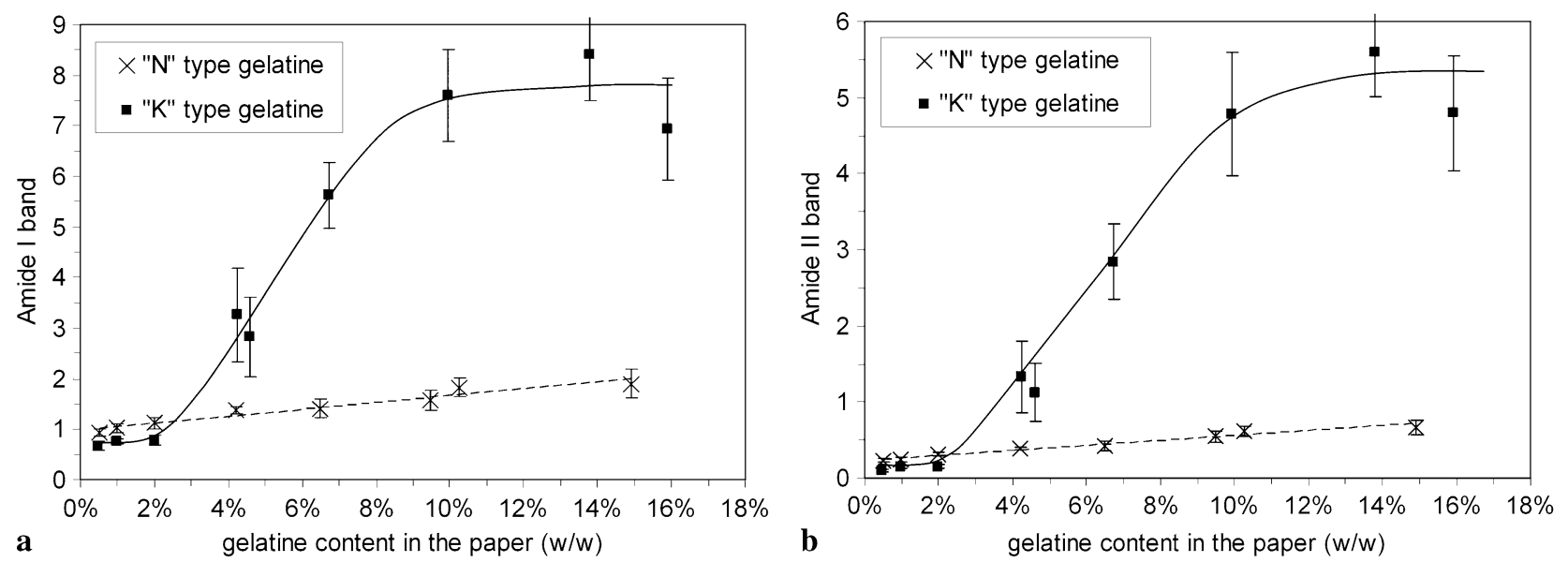

Fig. 3 Gelatine absorption band area versus the gelatine average content in the paper (a) amide I band; (b) amide II band. Error bars correspond to standard deviations

\subsubsection{FTIR microscopy measurements}

The possibilities offered by FTIR microscopy for obtaining information on the gelatine profile along the depth of the paper were investigated on two specific samples. The first, called "K-3\%", was impregnated with a $3 \%$ w/w solution of type "K" gelatine. The second, called "N-3\%", was impregnated with a $3 \% \mathrm{w} / \mathrm{w}$ solution of type " $\mathrm{N}$ " gelatine. Both samples contain approximately $6.9 \% \mathrm{w} / \mathrm{w}$ gelatine.

Two samples were collected from each paper, and a minimum of two transversal slices were prepared from each sample. FTIR maps recorded on these slices qualitatively show similar characteristics (Fig. 4). First, the higher the resin absorption, the lower the cellulose absorption. This indicates that the resin penetrates deep between the paper fibers, but not into the fibers themselves. Secondly, on "N-3\%" samples the gelatine signal is clearly superimposed on that of the cellulose, suggesting an even distribution of gelatine along the paper thickness. On the contrary, on "K-3\%" samples, the gelatine signal is higher near to the paper surface, suggesting a preferential surface deposition.

We then attempted to quantify more precisely the gelatine distribution within the paper. Lateral slices of the resinembedded sample were cut in a regular fashion, parallel to both surfaces, the slice number being related to the depth from the upper surface. On each slice, the average of 20 FTIR spectra was recorded. As the thickness of the slices is only controlled to within a precision of $\pm 25 \%$ by the microtome, we applied on each spectrum a correction factor corresponding to the inverse of the height of the resin absorption peak at $1735 \mathrm{~cm}^{-1}$. In the spectral region between $1800 \mathrm{~cm}^{-1}$ and $1500 \mathrm{~cm}^{-1}$ of the averaged and normalized spectra, a linear background correction was applied. The areas of the amide I $\left(1693 \mathrm{~cm}^{-1}\right.$ to $\left.1592 \mathrm{~cm}^{-1}\right)$ and amide II $\left(1592 \mathrm{~cm}^{-1}\right.$ to $\left.1504 \mathrm{~cm}^{-1}\right)$ bands were then calculated. The amide I band was evidently overestimated, as this band consists of a small peak near the large absorption peak of the resin $\left(1680 \mathrm{~cm}^{-1}\right.$ to $\left.1770 \mathrm{~cm}^{-1}\right)$. The absorption of the resin peak between $1693 \mathrm{~cm}^{-1}$ to $1592 \mathrm{~cm}^{-1}$ was then subtracted from the amide I area in order to obtain its net magnitude. This resin absorption in this spectral window was estimated from an average of 20 spectra that were recorded in similar conditions from a pure (resin-impregnated) Whatman paper sample, using a similar data processing.

The net amide I and amide II signals where then plotted versus the paper depth. On the "N-3\%" sample, these signals remained constant, confirming a uniform gelatine distribution (Fig. 5a). On the "K-3\%" sample, these signals were significantly decreasing in the inner part of the paper, confirming a gelatine gradient, easily described by means of a second-order polynomial function (Fig. 5b). Finally, the amide I and amide II signals, first expressed in arbitrary units, were converted into quantitative units (\% gelatine w/w per micrometer paper) by calculating the total area under the interpolated curve and considering that this area was corresponding to a global gelatine content $(6.9 \% \mathrm{w} / \mathrm{w})$. It should be noted that the net Amide I and Amide II depth profiles obtained in this manner are perfectly superimposing, confirming that the correction applied to obtain the net amide I band intensity was appropriate.

\subsubsection{Sizing properties}

The hydrophobic aspects of the paper samples were briefly evaluated by measuring the time necessary for the penetration of $0.1 \mu \mathrm{l}$ drop of water in the paper. This penetration was instantaneous in the case of "N-3\%" samples and took $200 \mathrm{~s} \pm 30 \mathrm{~s}$ in the case of "K-3\%" samples. This brief test shows that, despite similar average gelatine contents, these two samples are behaving very differently toward water. Exclusively considering the average gelatine content is obviously not enough to fully describe the sizing effect of gela- 


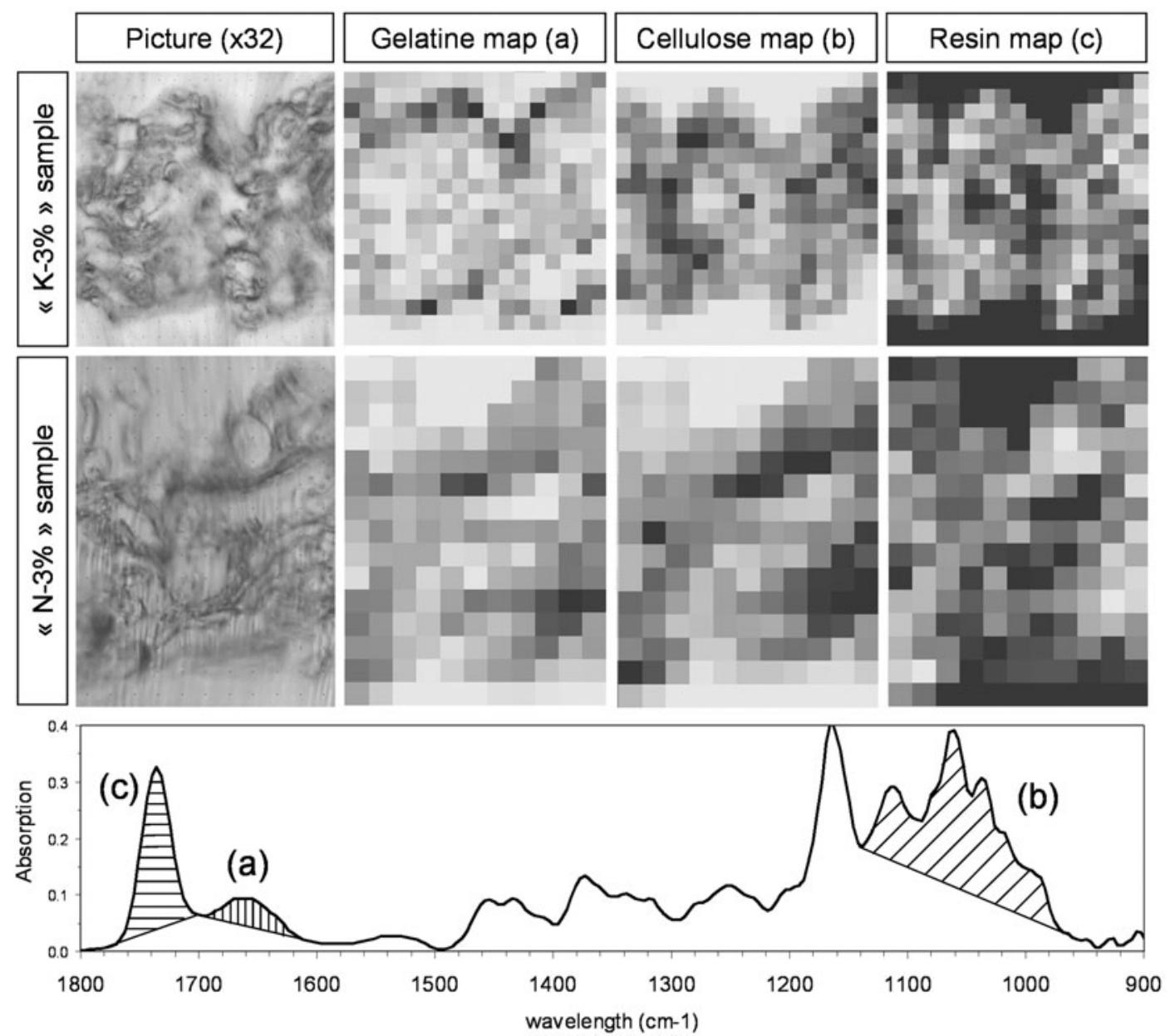

Fig. 4 Example of transversal slices FTIR mapping. Each pixel represents $10 \mu \mathrm{m} \times 10 \mu \mathrm{m}$. Below: an FTIR spectrum recorded on one of the samples. The (a), (b), and (c) areas respectively correspond to the gelatine, the cellulose, and the resin signal
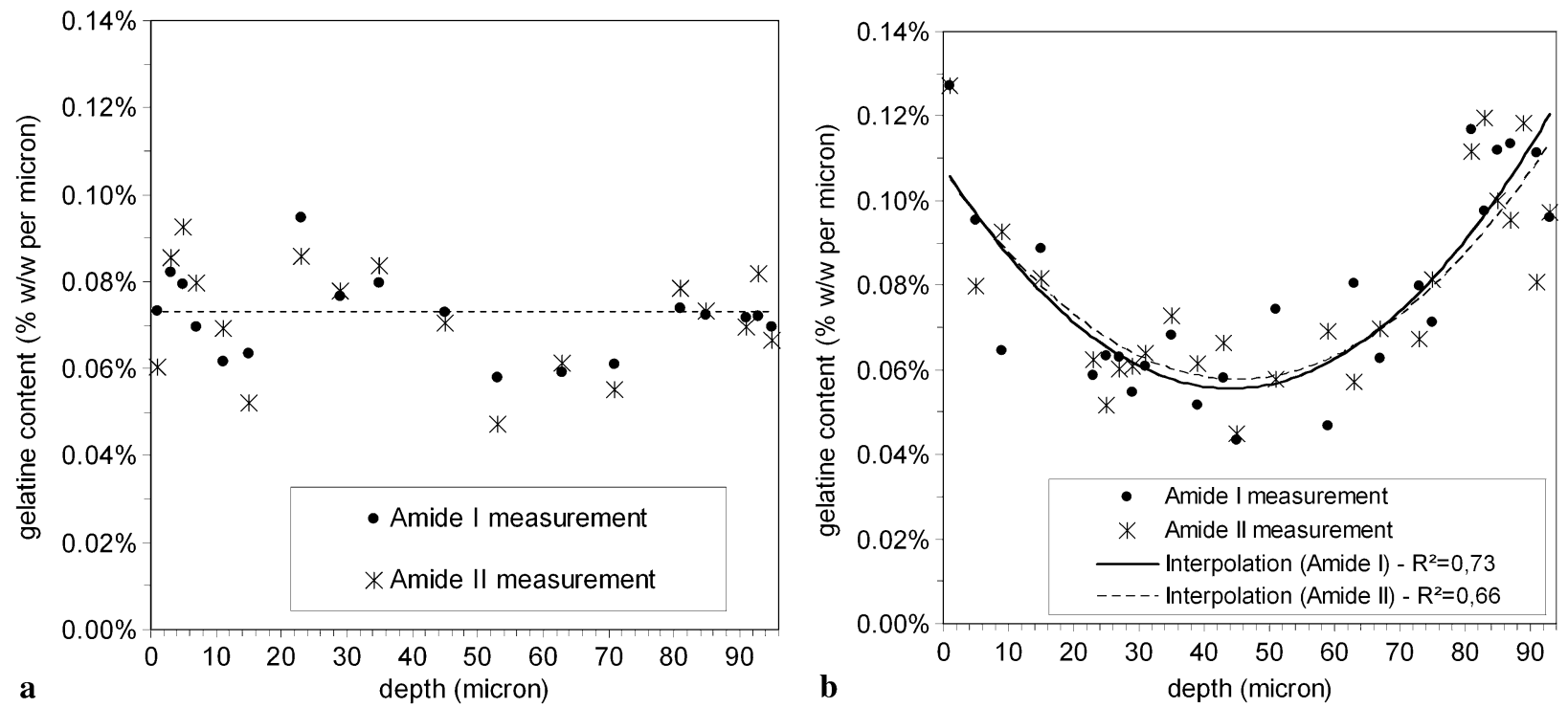

Fig. 5 Gelatine concentration profile in the paper. (a) "N" type gelatine; (b) "K" type gelatine 
tine. Many other parameters may have an impact, such as the size distribution within the paper or the type of gelatine to be used. This aspect may deserve a more detailed study in order to arrive at a better understanding of the sizing effect of gelatine, when used to restore original papers.

\section{Conclusion}

This work shows that FTIR-ATR spectroscopy is an efficient method to qualitatively test for the presence of gelatine in paper. However, it cannot be considered as a quantitative method to determine the gelatine content in paper as it is highly dependent on the gelatine distribution in the paper. FTIR microscopy appears to be a more efficient method for evaluating the gelatine content and its distribution along the thickness of the paper. FTIR mapping of transversal thin slices provides in first place some qualitative information on the evenness or unevenness of the gelatine distribution within the paper depth. However, it requires high performance equipment that makes use of synchrotron radiation, and this equipment is not easily accessible. A complementary approach consists in examining lateral slices, cut parallel to the paper surface with a conventional FTIR microscope, a type of instrument that is more easily accessible. This approach is more laborious with respect to sample preparation, because a large number of slices need to be prepared for the analysis of one sample, but it also consistently yields more quantitative information.

Acknowledgements This work was performed with the support of the Fondazione Cassa di Risparmio di Torino, who founded the postgraduate internship of Eleonora Pellizzi at the CRCC (Master dei talenti). It received the technical support of the SMIS line of the SOLEIL synchrotron (Saint Aubin, France). We are thankful to all our colleagues who helped us during our experiments: Anne Laurence Dupont for providing gelatine samples, Chakib Djediat (Museum National d'Histoire Naturelle, Paris) for his advice regarding the samples preparation, Christophe Sandt (Synchrotron SOLEIL) for his help and availability during our Beam time allocation, and Paul Dumas (Synchrotron SOLEIL) for his warm reception on the SMIS line.

\section{References}

1. K. Garlick, Book Pap. Group Annu. 5, 94-107 (1986)

2. C.H. Stephens, T. Barrett, P.M. Whitmore, J.A. Wade, J. Mazurek, M. Schilling, J. Am. Inst. Conserv. 47, 201-215 (2008)

3. T. Barrett, P. Lang, J. Waterhouse, in International Conference on Conservation and Restoration of Archival and Library Materials, Erice, 22-29 April 1996, vol. 2 (1996), pp. 605-624

4. T. Barrett, C. Mosier, in The Institute of Paper Conservation Conference, Manchester, 1-4 April 1992 (1992), pp. 207-213

5. TAPPI, T 504 om-89, Tappi Test Methods (Technical Association of the Pulp and Paper Industry, Atlanta, 1991)

6. T. Barrett, C. Mosier, J. Am. Inst. Conserv. 34, 173-186 (1995)

7. J.F. Waterhouse, T.D. Barrett, Tappi J. 74, 207-212 (1991)

8. T.D. Barrett, Pap. Conserv. 13, 3-108 (1989)

9. A.-L. Dupont, J. Chromatogr. A 950, 113-124 (2002)

10. A.-L. Dupont, Gelatine Sizing and Its Impact on the Degradation of Cellulose During Ageing (University of Amsterdam, Amsterdam, 2003)

11. M. Missori, M. Righini, A.-L. Dupont, Opt. Commun. 263, 289294 (2006)

12. T. Barrett, C. Mosier, Book Pap. Group Annu. 13, 5-8 (1994)

13. P. Spitzmueller, in The Institute of Paper Conservation Conference, Manchester, 1-4 April 1992 (1992), pp. 214-217

14. G. Kolbe, Restaurator 25, 26-39 (2004)

15. D.A. Prystupa, A.M. Donald, Polym. Gels Netw. 4, 87-110 (1996)

16. J.H. Muyonga, C.G.B. Cole, K.G. Duodu, Food Chem. 86, 325332 (2004)

17. P. Calvini, A. Gorassini, Restaurator 23, 48-66 (2002) 